\title{
Speaker Separation and Tracking System
}

\author{
U. Anliker, J. F. Randall, and G. Tröster
}

The Wearable Computing Lab, ETH Zurich, 8097 Zurich, Switzerland

Received 26 January 2005; Revised 5 December 2005; Accepted 8 December 2005

\begin{abstract}
Replicating human hearing in electronics under the constraints of using only two microphones (even with more than two speakers) and the user carrying the device at all times (i.e., mobile device weighing less than $100 \mathrm{~g}$ ) is nontrivial. Our novel contribution in this area is a two-microphone system that incorporates both blind source separation and speaker tracking. This system handles more than two speakers and overlapping speech in a mobile environment. The system also supports the case in which a feedback loop from the speaker tracking step to the blind source separation can improve performance. In order to develop and optimize this system, we have established a novel benchmark that we herewith present. Using the introduced complexity metrics, we present the tradeoffs between system performance and computational load. Our results prove that in our case, source separation was significantly more dependent on frame duration than on sampling frequency.
\end{abstract}

Copyright ( 2006 Hindawi Publishing Corporation. All rights reserved.

\section{INTRODUCTION}

The human ability to filter competing sound sources has not been fully emulated by computers. In this paper, we propose a novel approach including a two-step process to automate this facility. The two-step process we propose is based on combining speaker separation and speaker tracking into one system. Such a system could support transcription (words spoken to text) of simultaneous and overlapping voice streams individually. The system could also be used to observe social interactions.

Today, speaker tracking and scene annotation systems use different approaches including a microphone array and/or a microphone for each user. The system designer typically assumes that no overlap between speakers in the audio stream occurs or segments with overlap are ignored. For example, the smart meeting rooms at Dalle Molle Institute [1] and Berkeley [2] are equipped with microphone arrays in the middle of the table and microphones for each participant. The lapel microphone with the highest input signal energy is considered to be the speaker to analyze. For this dominant speaker, the system records what has been said. Attempts to annotate meetings [3] or record human interactions [4] in a mobile environment have been presented, both systems assumed nonoverlapping speech in the classification stage.

Each speech utterance contains inherent information about the speaker. Features of the speakers voice have been used to annotate broadcasts and audio archives, for example, [5-7]. If more than one microphone is used to record the scene, location information can be extracted to cluster the speaker utterances, for example, $[8,9]$. The work of Ajmera et al. [10] is to our knowledge the first which combines location and speaker information. Location information is extracted from a microphone array and speaker features are calculated from lapel microphones. An iterative algorithm summarizes location and speaker identity of the speech segments in a smart meeting room environment. Busso et al. [11] presented a smart meeting room application by which the location of the participants is extracted from video and audio recordings. Audio and video locations are fused to an overall location estimation. The microphone array is steered towards the estimated location using beamforming techniques. The speaking participant and his identity are obtained from the steered audio signal.

The goal of our work is to develop a system which can be used outside of specially equipped rooms and also during daily activities, that is, a mobile system. In order for such a mobile system to be used all day, it has to be lightweight $(<100 \mathrm{~g})$ and small $\left(<30 \mathrm{~cm}^{3}\right)$. Such size and weight constraints limit energy, computational resources, and microphone mounting locations. An example of a wearable computer in this class is the QBIC (belt integrated computer) that consumes $1.5 \mathrm{~W}$ at 400 MIPS [12]. This system can run for several hours on one battery. To tailor the system design to low power, we propose a benchmark metrics which considers the computational constraints of mobile computing.

The vision of wearable systems is a permanently active personal assistant $[13,14]$. The personal assistant provides instantaneous information to the wearer. In the context of a speaker tracking system, the instantaneous information can 


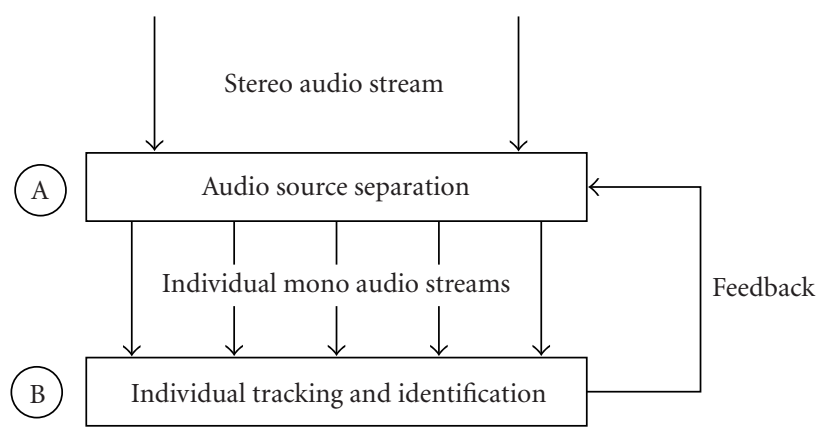

FIgURE 1: Two-step process to track individuals. (A) Source separation. (B) Tracking and identification.

influence and improve the course of a discussion. For example, a message could indicate when a maximal speech duration threshold has been reached, or a teacher could be informed that he/she has been speaking the majority of the time.

If a system has to be effective outside of the especially equipped rooms, it has to cope with conversational speech. Investigations by Shriberg et al. [15] showed that overlap is an important, inherent characteristic of conversational speech that should not be ignored. Also, participants may move more freely during a conversation. Equally, the environmental parameter may change, for example, room transfer function. A mobile system must be capable of adjusting to these parameter changes.

We opted to use two microphones as they can be unobtrusively integrated into clothing and mobile devices and as we seek to replicate the human ability to locate audio sources with two ears. The clothing has to be designed in such a way that the relative position of the two microphones does not vary due to movements. Additionally, a rotation sensor is required to compensate the changes of body orientation-the compensation will be integrated during further algorithm development. Employing a mobile device, the requirements of the microphone position can be easier satisfied as the microphone spacing is fixed and the mobile device can be placed on the table.

The audio data is recorded as a stereo audio stream, for example, each of the two microphones is recorded as one of the two stereo channels. The stereo audio stream is treated in the two-step process shown in Figure 1. First, the audio data is separated into individual audio streams for each detected source, respectively, speaker (A); then for each of these streams, individuals are tracked and identified (B). Step (B) may support step (A) by providing a feedback, for example, by providing information as to which location of an individual can be expected. Also, the location information (step (A)) can be used to bias the identifier (step (B)), for example, the individuals in the speaker database may not have an equal a priori probability.

To compare different system configurations, we introduce a benchmark methodology. This benchmark is based on performance metrics for each of the two steps ((A) and (B)).
We apply the concept of recall and precision [16] as a metrics to measure the system accuracy. Given that we target a mobile system, we also introduce a complexity factor that is proportional to the use of computational resources as metrics to measure the system energy consumption. The benchmark metrics and the system performance are evaluated with experiments in an office environment. The experiments point out the influence of microphone spacing, time frame duration, overlap of time frames, and sampling frequency.

The novelty of this paper can be found in a number of areas. Firstly, a system is presented that combines speaker separation and tracking. In particular, a feedback loop between speaker separation and speaker tracking is introduced and optimal system parameters are determined. Secondly, the system addresses the speaker tracking problem with overlap between different sound sources. Thirdly, a mobile system is targeted, for example, only limited system resources are available and the acoustical parameters are dynamic. Finally, a novel benchmark methodology is proposed and used to evaluate accuracy and computation complexity. Computation complexity has not been previously used as a design constraint for speaker separation and tracking systems.

A description of the implemented system and of the three tuning parameters is given in Section 2. In Section 3, we present the benchmark methodology for the two-step speaker separation and tracking system. The experimental setup and simulation results are presented in Section 4. These show that recall and precision of the separation are independent of sampling frequency, but depend on the time frame duration. We also show that the feedback loop improves recall and precision of the separation step (step (A)).

\section{SYSTEM DESCRIPTION}

In this section, we present our implementation of the twostep system shown in Figure 1. The complete signal flow in Figure 2 includes a preprocessing step. The preprocessing step reduces signal noise and enhances the higher spectrum components to improve speaker recognition performance. A bandpass filter ([75 7000] Hz) and preemphasize filter $\left(1-.97 z^{-1}\right)$ are applied to each of the two input audio streams. In the first processing step (A) the different audio sources are separated. The implemented blind source separation (BSS) algorithm is based on spatial cues. The audio data having the same spatial cues are clustered to an audio stream. In the second step (B), individuals are tracked and identified for each audio stream. To each individual audio stream three tasks are applied. First, the audio stream is split into speech and nonspeech frames. Then the speech frames are analyzed for speaker changes. Lastly, the data between two speaker change points and/or between speech bounds is used to identify a speaker.

\subsection{Blind source separation (step (A))}

A source separation algorithm has to fulfill the following two criteria to be suitable for our proposed system. First, the algorithm has to cope with more sources than sensors. The 


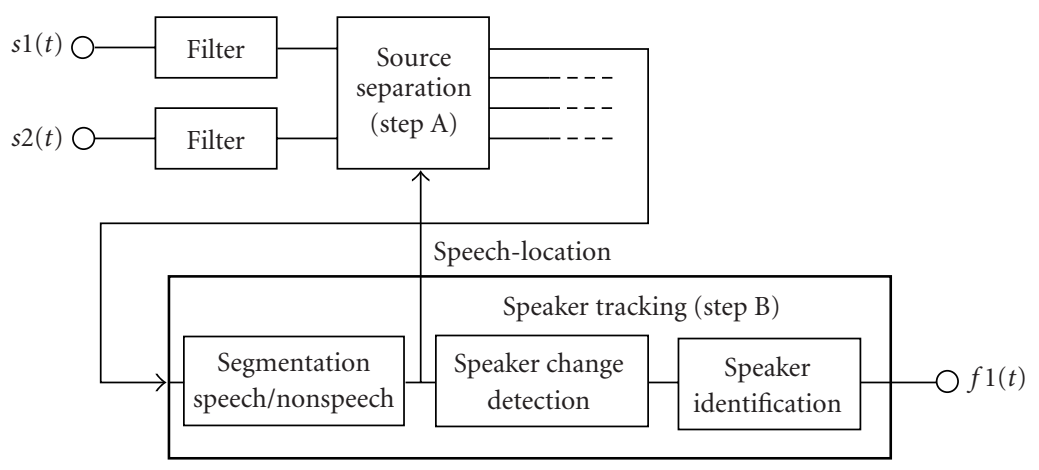

FIgUre 2: Data flow: the input data stream $s 1(t)$ and $s 2(t)$ is first filtered. A BSS algorithm splits the input stream into a data stream for each active audio source based on spatial cues (step (A)). Step (B): for each source location the data is segmented in speech and nonspeech segment. The speech segments are analyzed for speaker changes and later a speaker identity is assigned.

separation problem is degenerated and traditional matrix inversion demixing methods cannot be applied. Second, the system has to provide an online feedback to the user. The algorithm has to be capable of separating the data and producing an output for each audio source for every time segment. The sound source location can be employed to cluster the audio data and to bias the speaker identifier. Therefore, algorithms that provide location information directly are favored. The degenerate unmixing estimation technique (DUET) algorithm [17] fulfills the above criteria. The algorithm performance is comparable to other established blind source separation (BSS) methods [18]. One of the separation parameters is the time difference of arrival (TDOA) between the two microphones. We give a description of the DUET algorithm and introduce two of our modifications.

The input signal is a stereo recording of an audio scene $\left(X_{1}(t)\right.$ and $\left.X_{2}(t)\right)$. The input data stream is split into overlapping time frames. For each time frame the short time Fourier transformation (STFT) for both channels, $\mathbf{X}_{\mathbf{1}}[k, l]$ and $\mathbf{X}_{2}[k, l]$, is computed. Based on the STFT, the phase delay

$$
\tilde{\delta}[k, l]=-\frac{m}{2 \pi k} \angle \frac{\mathbf{X}_{2}[k, l]}{\mathbf{X}_{\mathbf{1}}[k, l]}
$$

and the amplitude ratio

$$
\tilde{\boldsymbol{\alpha}}[k, t]=\left|\frac{\mathbf{X}_{2}[k, l]}{\mathbf{X}_{\mathbf{1}}[k, l]}\right|
$$

between the two channels are calculated. $m$ is the STFT length, $k$ the frequency index, and $l$ the time index. $\angle$ denotes the argument of the complex number. The data of a particular source has a similar phase delay and amplitude ratio.

The time frames are grouped into time segments. For each time segment, that is, the frame group, a 2D histogram is built. One direction represents the amplitude ratio and the other the phase delay. We expect that the bins of the 2D histogram corresponding to a phase delay and an amplitude ratio of one source will have more data points and/or signal energy than others. Thus, each local maximum in the histogram represents an audio source. Each $(\tilde{\boldsymbol{\delta}}[k, i], \tilde{\boldsymbol{\alpha}}[k, i])$ data point is assigned to one of the local maximums, that is, source, by a maximum likelihood (ML) estimation. The algorithm assumes that the sources are pairwise $\mathrm{W}$-disjoint orthogonal, that is, each time-frequency point is occupied by one source only. The W-disjoint orthogonality is fulfilled by speech mixtures insofar as the mixing parameter can be estimated and the sources can be separated [19].

Our first experiments demonstrated two issues. First, in the presence of reverberation (e.g., as in office rooms) the performance of the DUET algorithm degenerates. Second, if the two microphones are placed close together-compared to the distance between the sources and the microphonesthe amplitude ratio is not a reliable separation parameter. To address these two issues, the DUET algorithm is modified. First, a 400 -bin $1 \mathrm{D}$ histogram based on $\tilde{\boldsymbol{\delta}}[k, i]$ is employed. The histogram span is evenly distributed over twice the range of physical possible delay values. The span is wider than the physical range as some estimations are expected to be outside the physical range. Second, the implementation uses the number of points in each bin and not a power-weighted histogram as suggested in [17]. Data points which lie in a specified frequency band are considered, see next paragraph. To reduce the influence of noise, only data points that have a total power of $95 \%$ of the frequency band power in one time segment are taken into account. We refer to this modified DUET implementation as DUET-PHAT (phase transform). The modifications are deduced from the single-source case. For a single-source TDOA estimation by generalized cross correlation (GCC), several spectral weighting functions have been proposed. Investigation on uniform cross-correlation (UCC), ML, and PHAT time-delay estimation by Aarabi et al. [20] showed that, overall, the PHAT technique outperformed the other techniques in TDOA estimation accuracy in an office environment.

Basu et al.[21] and others showed that the full signal bandwidth cannot be used to estimate the delay between two microphones. The phase shift between the two input signals needs to be smaller than $\pm \pi$. If signal components exist that have a shorter signal period than twice the maximal 
delay, bigger phase shifts can occur and the BSS results are no longer reliable. For our configuration (microphone spacing of $10 \mathrm{~cm}$ ) this minimal signal period is 0.059 millisecond, which is equivalent to a maximal frequency $f_{\text {crit }}$ of $1.7 \mathrm{kHz}$. Increasing the low-pass filter frequency above $1.7 \mathrm{kHz}$ has two effects. Firstly, the delay accuracy in the front region (small delay) is increased. Secondly, delay accuracy at the sides is reduced, see Section 4.1.2. We decided to use a [200 $3400] \mathrm{Hz}$ digital bandpass filter for the blind source separation step for two reasons. First, the maximum energy of average long-term speech spectrum (talking over one minute) lies in the $250 \mathrm{~Hz}$ and the $500 \mathrm{~Hz}$ band. Second, we expect most individuals to be in front of the microphones.

If speakers and/or the system moves, the mixing parameters will change. To cope with dynamic parameters, the time segments have to be short. Our experiments showed that a time segment duration of $t_{\text {seg }}=1.024$ seconds is suitable to separate sources as recall is above 0.85 ; for information $t_{\text {seg }}=0.512$ second has a recall of 0.50 . The identification trustworthiness is correlated to the amount of speaker data. To increase the speaker data size our algorithm tracks the speaker location. If the speaker location has changed less than dis $_{\max }=0.045$ millisecond since the last segment, we assume that the algorithm has detected the same speaker. Therefore, a speaker moving steady-going from the left side (view angle -90 degrees) to the right side (view angle +90 degrees) in 20 seconds can be theoretically followed. Our experiments showed a successful tracking if the speaker took 60 seconds or more.

\subsection{Speaker tracking (step (B))}

The data with similar delay and thus with similar location are clustered into one data stream. Each stream can be thought of as a broadcasting channel without any overlaps. Systems to detect speaker changes and to identify the speakers have been presented by several research groups, see [5-7, 22]. In the next paragraphs, we present our implementation.

The audio data is converted to 12 MFCCs (mel-frequency cepstrum coefficients) and their deltas. The MFCCs are calculated for each time frame. The annotation of the audio stream is split into three subtasks. First, the audio stream is split into speech and nonspeech segments. Second, the speech segments are analyzed for speaker changes. An individual speaker utterance is then the data between two speaker change points and/or between speech segment bounds. Third, the speaker identity of an individual utterance is determined, that is, the data from a single utterance is employed to calculate the probability that a particular person spoke. If the normalized probability is above a preset threshold, then the utterance is assigned to the speaker with the highest probability. If the maximum is below the threshold, a new speaker model is trained. Overall, the speaker tracking algorithm extracts time, duration, and the number of utterances of individual speakers. Intermediate results can be shown or recorded after each speaker utterance.

\subsubsection{Speech/nonspeech detection}

Only data segments that are comprised mainly of speech can be used for identifying an individual speaker. Nonspeech segments are therefore excluded. The most commonly used features for speech/nonspeech segmentation are zero-crossing rate and short-time energy, for example, see [23]. The input data is separated and clustered in the frequency domain. Thus, it is computationally advantageous to use frequency domain features to classify the frames. Spectral fluctuation is employed to distinguish between speech and nonspeech. Peltonen et al. used this feature for computational auditory scene analysis [24] and Scheirer and Slaney for speech-music discrimination [25].

\subsubsection{Speaker change}

The goal of the speaker change detection algorithm is to extract speech segments, during which a single individual is speaking, that is, split the data into individual speaker utterances. The signal flow of the speaker change detection algorithm is shown in Figure 3. The algorithm is applied to the speech data of an utterance, that is, the nonspeech data is removed from the data set. If for more than $t_{\text {pause }}=$ 5 seconds nonspeech segments are detected or if for more than $t_{\text {speaking }}=15 \mathrm{~s}$ an utterance is going on, then the speaker change detection and identification is executed. To reduce the influence of the recording channel, CMS (cepstral mean substraction) [26] is applied.

Depending on the data size, two different calculation paths are taken. If the speech data size is smaller than $t_{1}=$ 2.048 seconds, the data is compared to the last speech segment. The two data sets are compared by the Bayesian information criterion (BIC) [27]:

$$
\begin{aligned}
\Delta \mathrm{bic}= & -\frac{n_{1}}{2} \log \operatorname{det}\left(\mathbf{C}_{\mathbf{1}}\right)-\frac{n_{2}}{2} \log \operatorname{det}\left(\mathbf{C}_{2}\right) \\
& +\frac{n}{2} \log \operatorname{det}(\mathbf{C})+\lambda \cdot p \\
p= & \frac{1}{2}\left(d+\frac{d(d+1)}{2}\right) \log (n)
\end{aligned}
$$

where $n_{1}$ and $n_{2}$ are the data size of first and second data segment, respectively, the overall data size $n=n_{1}+n_{2}$. $\mathbf{C}, \mathbf{C}_{1}$, and $\mathbf{C}_{2}$ are the diagonal covariance matrix estimated on the data set. $d$ is the data dimensionality. $\lambda$ is the penalty weight, we use 1 . If the $\Delta$ bic value is above zero, this means that no speaker change occurred and the speech segment is given the same speaker identification as the last one. If $\Delta$ bic is below zero, then a speaker change is assumed and the speaker identification module is called.

For long speech segments, the algorithm checks for internal speaker changes. The speaker change detection has three sequential processes with each confirming the findings of the previous process. The first process is based on the comparison of two adjacent segments of $t_{1} / 2$ duration. A potential speaker change point is equal to the local maximum of the distance measure $D$. The data segments are represented by a 


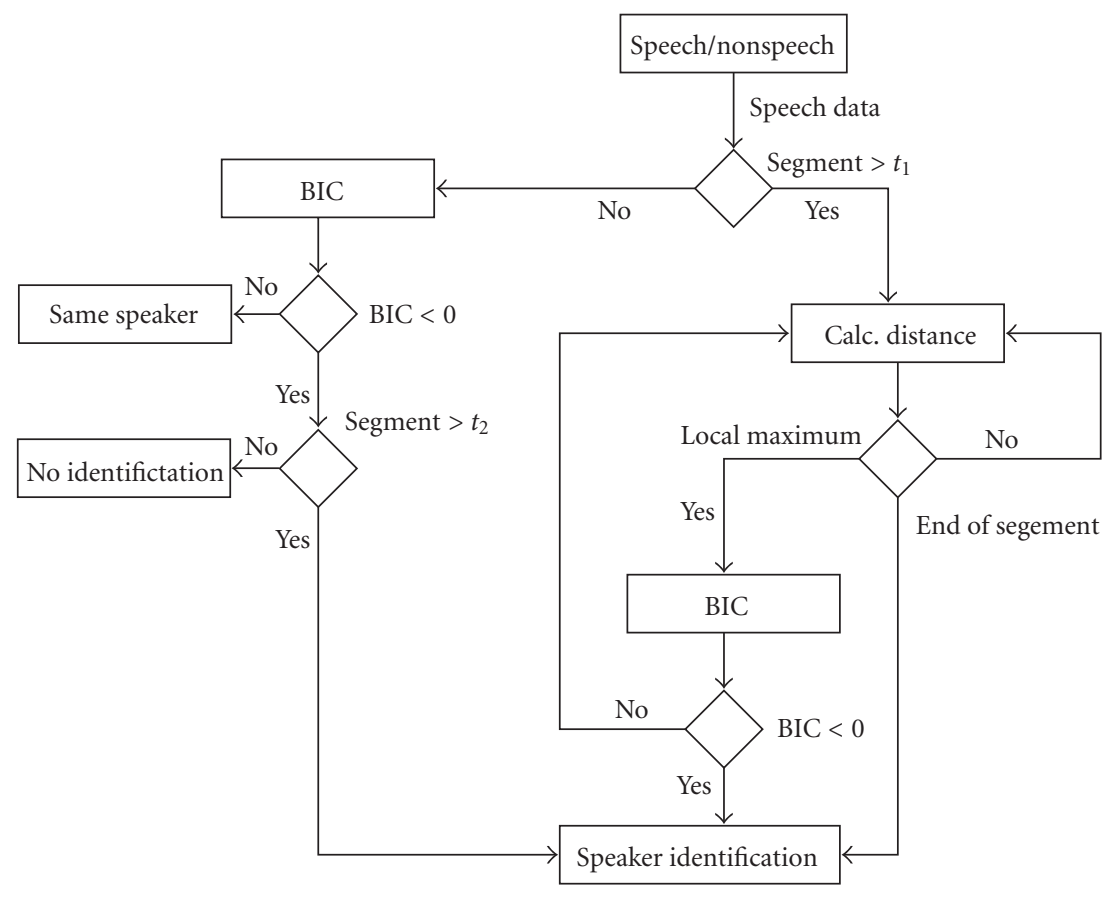

FIGURE 3: Speaker change detection: for short segments a speaker identification is performed directly. Long speaker segments are checked for intraspeaker changes. Consequently, the identifier is run on homogeneous data segments.

unimodal Gaussian mixture with diagonal covariance matrix C. The distance $D$ is calculated by [7]

$$
D(i, j)=\frac{1}{2} \operatorname{tr}\left(\left(\mathbf{C}_{\mathbf{i}}-\mathbf{C}_{\mathbf{j}}\right)\left(\mathbf{C}_{\mathbf{i}}^{-1}-\mathbf{C}_{\mathbf{j}}^{-1}\right)\right),
$$

$\operatorname{tr}$ is the matrix trace. A potential speaker change is found between $i$ th and $(i+1)$ th segment, if the following conditions are satisfied: $D(i, i+1)>D(i+1, i+2), D(i, i+1)>D(i-1, i)$, and $D(i, i+1)>t h_{i}$, where $t h_{i}$ is a threshold. The threshold is automatically set according to the previous $s=4$ successive distances:

$$
t h_{i}=\alpha \frac{1}{s} \sum_{n=0}^{s} D(i-n-1, i-n),
$$

$\alpha$ is set to 1.2 . The segment is moved by 0.256 second. This is equivalent to the speaker change resolution.

The second process validates the potential speaker changes by the Bayesian information criterion (BIC), for example, as in [28-30]. If the speaker change is confirmed or the end of the speech segment is reached, the utterance speaker is identified.

In the third process of the speaker change detection the speaker identification can be seen. The implementation is described in the next section. For two adjacent utterances the same speaker can be retrieved. Only if a speaker change is confirmed by all three processes a new speaker is retrieved.

\subsubsection{Speaker identification}

A speaker identification system overview including the recognition performance can be found in [31]. For conver- sational speech, the speaker identifier has to deal with short speech segments, unknown speaker identities (i.e., no pretraining of the speaker model is possible), unlimited number of speakers (i.e., the upper limit is not known beforehand), and has to provide online feedback (i.e., the algorithm cannot work iteratively). We have implemented an algorithm based on a world model, which is adjusted for individual speakers.

The individual speakers are represented by a Gaussian mixture model (GMM) employing 16 Gaussians having a diagonal covariance matrix. We employ 16 Gaussians as investigation by [32-34] showed that starting from 16 mixtures a good performance is possible, even if only few feature sets can be used to train the speaker model. The model input features are 12 MFCCs and their deltas.

To identify the speaker of a speech utterance, the algorithm calculates the log likelihood of the utterance data for all stored speaker models. All likelihoods are normalized by a world model log likelihood and by the speech segment duration. If the normalized likelihoods $(\Lambda)$ are above a predefined threshold $t h_{\text {like }}$, then the speech segment is assigned to the model with the maximum likelihood [35]:

$$
\bigwedge(X)=\frac{\log p\left(X \mid \lambda_{\text {speaker }}\right)-\log p\left(X \mid \lambda_{\text {world }}\right)}{n_{\text {seg }}}
$$

$X$ is the input data, $\lambda_{\text {speaker }}$ the speaker model, $\lambda_{\text {world }}$ the world model, and $n_{\text {seg }}$ the number of time frames. If the likelihoods are below the threshold, then a new speaker model is trained using the world model as a seed for the EM (expectation maximization) algorithm. 


\subsection{Feedback speech segments to BSS}

Based on the speech/nonspeech classification, it is known at which location (represented by the delay) an individual is talking. If at the end of a time segment an individual is talking (for each time segments the audio sources are separated), then in the next time segment it is expected to detect an individual at the same location (expected locations).

The DUET-PHAT algorithm detects active sources, that is, speakers, as local maximums in the delay histogram (detected locations), see Section 2.1. All speakers cannot be detected in every segment, as the delay of a speaker can be spread, for example, by movements or noise, or as the local maximum is covered by a higher maximum.

The feedback loop between speaker tracking and BSS compares the detected locations by the BSS and the expected locations. A correspondence between expected and detected locations is found, if the difference between the two delays is smaller than dis $s_{\max }$. If no correspondence for an expected location is found, the delay is added to the detected locations. The data points are assigned to one of the detected delays or to the added delays by an ML estimation as without the feedback loop.

\subsection{Parameters}

Our two-step speaker separation and tracking system is controlled by more than 20 parameters. Most of them influence only a small part of the system, but if they are set incorrectly, the data for the following processing step is useless. The values used are mentioned in the text. The influences of the following four parameters are investigated one at a time in the experiment in Section 4, keeping all other parameters constant.

\subsubsection{Microphone spacing}

Placing the two microphones close together gives a high signal bandwidth which can be employed to estimate the source location, see Section 2.1. On the other hand, the requirements on the delay estimation precision are increased.

\subsubsection{Time frame duration}

For each time frame the STFT is calculated. Low frequency signals do not have a complete period within a short time frame, which leads to disturbance. It is then not possible to calculate a reliable phase estimation. The upper bound of the time frame duration is given by the assumption of quasistationary speech. The assumption is fulfilled up to several tens of milliseconds and fails for more than 100 milliseconds [36]. The time frame duration $\left(t_{\text {frame }}\right)$ determines the frequency resolution $\left(f_{\text {res }}\right)$ :

$$
f_{\text {res }}=\frac{f_{\text {sample }}}{m}=\frac{f_{\text {sample }}}{f_{\text {sample }} t_{\text {frame }}}=\frac{1}{t_{\text {frame }}},
$$

where $f_{\text {sample }}$ is the sampling frequency and $m$ the number of points in the STFT. Investigations by Aoki et al. [37] showed that a frequency resolution between 10 and $20 \mathrm{~Hz}$ is suitable to segregate speech signals. The percentage of frequency components that accumulate $80 \%$ of the total power is then minimal. Aoki et al. showed that for a frequency resolution of $10 \mathrm{~Hz}$, the overlap between different speech signals is minimal. Baeck and Zölzer [38] showed that the Wdisjoint orthogonality is maximal for a 4096-point STFT, when using $44.1 \mathrm{kHz}$ sampling frequency, which is equivalent to a frequency resolution of $10.77 \mathrm{~Hz}$. We expect to get best separation results for a time frame duration between 50 milliseconds and 100 milliseconds. The time frame duration typically used in speech processing is shorter. The duration is in the range of 10 milliseconds to 30 milliseconds.

\subsubsection{Time frame shift}

The time frame shift defines to what extent the time frame segments overlap. If the shift is small, more data is available to train a speaker model and more time-frequency points can be used to estimate the source position. However, the computation complexity is increased.

\subsubsection{Sampling frequency}

The delay estimation resolution is proportional to the sampling frequency. Increasing the sampling frequency increases the computation complexity.

\section{BENCHMARK}

As we have a two-step system (Figure 1) we opted for a twostep benchmark methodology; a further reason for such an approach is that the performance of step (B) depends on the performance of step (A).

In designing the benchmark, the following two cases have to be taken into account. The first is that only sources detected during the separation step can be later identified as individuals. The second issue is that if too many sources are detected, three different outcomes are possible. In the first outcome, a noise source is detected which can be eliminated by a speech/nonspeech discriminator. In the second outcome, an echo is detected, which will be considered as separate individual or the identification allows the retrieval of the same individual several times in the same time segment, then a merging of these two to one is possible. In the third outcome, depending on the room transfer function and noise, nonexistent artificial sources can be retrieved that will collect signal energy from the true sources. These outcomes will impact the performance of the identification step.

In order to cope with the dependance between the two steps, the system is first benchmarked for step (A) and then for both steps $(A$ and $B$ ) including the feedback loop $(B \rightarrow A)$. For both steps, we define an accuracy measure to quantify the system performance. The measures are based on recall and precision. Ground truth is obtained during the experiments by a data logger that records the start and stop time of a speech utterance and the speaker location. 
As mobile and wearable systems usually run on batteries, a strict power budget must be adhered to. During system design, different architectures have to be evaluated with the power budget in mind. Configurations that consume less system resources are favored. A second optimization criterion deals with the system power consumption. During the algorithm development, we assume a fixed hardware configuration. The energy consumption is therefore proportional to the algorithm complexity. We introduce a relative complexity measure which reflects the order and ratio of the computation complexity.

\subsection{Accuracy}

We introduce for step (A) an accuracy metrics which reflects how well sound sources have been detected. The overall system metrics reflects how well individual speakers are identified and tracked. We selected an information retrieval accuracy metrics [16] as this metrics is calculated independently of the number of sources, is intuitive, and the ground truth can be recorded online.

\subsection{1. $\operatorname{Step}(A)$}

The implemented separation algorithm estimates for each segment and each source the signal delay between the two microphones. The delay estimation is defined as correct if the difference between the true delay and estimated one is below a preset tolerance.

Recall (rec) is defined as the number of segments in which the delay is estimated within a preset tolerance to the ground truth divided by the total number of active segments of the source. If more than one source is active, then the minimal recall rate is of interest. For example, if two sources are active, one source is detected correctly and the second one not at all, the average recall rate is 0.5 and the minimum 0.0 . The signal of the second source is then erroneously assigned to the detected one. Indeed, the audio data is not separated. The speaker identification has to be accomplished with overlapping speech, which is not possible.

Precision (pre) is defined as the number of correctly estimated delays (difference between the estimation and the ground truth is smaller than a preset tolerance) divided by the total number of retrieved delay estimations. An overall precision is calculated. In the multisource case, retrieved delays may belong to any of the active sources. Estimations which differ more than the preset tolerance to any source are considered as erroneous.

Precision and recall values are combined into a single metrics using the F-measure. The F-measure is defined as [39]

$$
f=2 \frac{\mathrm{rec} \cdot \mathrm{pre}}{\mathrm{rec}+\mathrm{pre}}
$$

To summarize, recall is equal to one if in all time segments all sources have been detected. If no sources are detected, then recall is zero. Precision is unity if no sources are inserted, and decreases towards zero as more nonexisting
TABLE 1: Definition of the confusion matrix for our experiments: rows represent the ground truth speaker and columns the retrieved speakers. SP $R_{i}$ is the $i$ th retrieved speaker, and SP $T_{j}$ is the $j$ th ground truth speaker.

\begin{tabular}{|c|c|c|c|}
\hline \multirow{2}{*}{$\begin{array}{l}\text { Ground truth } \\
\text { speaker }\end{array}$} & \multicolumn{3}{|c|}{ Speaker retrieved } \\
\hline & $\mathrm{SP} R_{1}$ & $\ldots$ & $\mathrm{SP} R_{n}$ \\
\hline $\mathrm{SP} T_{1}$ & $\frac{\mathrm{SP} R_{1} \text { when SP } T_{1}}{\text { Duration of SP } T_{1}}$ & $\cdots$ & $\frac{\text { SP } R_{n} \text { when SP } T_{1}}{\text { Duration of SP } T_{1}}$ \\
\hline $\mathrm{SP} T_{m}$ & $\frac{\text { SP } R_{1} \text { when SP } T_{m}}{\text { Duration of SP } T_{m}}$ & $\cdots$ & $\frac{\text { SP } R_{n} \text { when SP } T_{m}}{\text { Duration of SP } T_{m}}$ \\
\hline
\end{tabular}

sources are detected. For a flawless working system, recall and precision are equal to one. If many nonexisting sources are inserted, precision is low and the signal energy is distributed among the inserted sources. None of the sources will represent a speaker as the speaker features are split between the erroneously detected audio sources. The speaker identification cannot identify any speaker reliably. If many sources are not detected, recall is low and the signal energy is linked to a wrong source. The speaker features of two or more speakers are then combined to one. Possibly the dominant speaker might be detected, that is, the speaker with the highest signal energy, or a new speaker is retrieved.

\subsection{2. $\operatorname{Step} A+B$}

This system accuracy metrics has to reflect how well individual speakers are identified and tracked. We compare time segments assigned to one individual with the ground truth.

The system recall $\left(\mathrm{rec}_{\mathrm{sys}}\right)$ is defined as the duration of correctly assigned speech segments divided by the total duration of the speech. If for one time segment several speakers are retrieved, then the segment is counted as correct if the speaker has been retrieved at least once.

The system false rate $\left(\mathrm{fal}_{\mathrm{sys}}\right)$ is the duration of data which has been assigned erroneously to one speaker divided by the total retrieved speech duration. If in one time segment the correct speaker has been retrieved more than once, the time for the second retrieval is also considered to be assigned correctly, that is, we allow the same speaker to be at different locations in one time segment. The system is not penalized for detecting echoes.

To get a deeper insight of the system accuracy, we introduce a confusion matrix, see Table 1 . Rows represent the speaker ground truth. Each column represents a retrieved speaker. It is possible for more or less speakers to be retrieved than there are ground truth speakers since the number of speakers is determined online by the algorithm. For each ground truth speaker (row), the time assigned to a retrieved speaker (column) is extracted and then divided by the total speech time of the ground truth. The correspondence between retrieved speaker and ground truth speaker is calculated as follows: the maximal matrix entry (retrieved speaker time) is the first correspondence between ground truth (row index) and retrieved speaker (column index). Row and column of this maximum are removed from the matrix. The next correspondence is the maximal matrix entry of the 
TABLE 2: Sampling frequency complexity factor.

\begin{tabular}{l|cc}
\hline Frequency & Application & Factor \\
\hline $08 \mathrm{kHz}$ & Telephone line & 1 \\
$16 \mathrm{kHz}$ & Video conference, G.772 & 2 \\
$22.05 \mathrm{kHz}$ & Radio & 2.76 \\
$32 \mathrm{kHz}$ & Digital radio & 4 \\
$44.1 \mathrm{kHz}$ & $\mathrm{CD}$ & 5.51 \\
\hline
\end{tabular}

TABLE 3: Approximation of the STFT complexity factor.

\begin{tabular}{cc}
\hline No. of points & Factor \\
\hline 128 & 1 \\
256 & 0.75 \\
512 & 0.62 \\
1024 & 0.56 \\
2048 & 0.53 \\
4096 & 0.52 \\
\hline
\end{tabular}

remaining matrix. These steps are repeated until the matrix is empty.

\subsection{Computation complexity}

During the algorithm optimization, we assume that the hardware configuration is fixed. The energy consumption is then proportional to the computation load. The computation load can be defined in terms of elementary operations or classes of elementary operations as in [40]. If complex algorithms are developed in a high-level environment, such as Matlab, then it is a nontrivial task to estimate the number of elementary operations. Furthermore, during development it is not essential to know the absolute values, for example, run time, as these depend on the computation platform and on the optimization techniques applied. To guide a design decision, it is sufficient to know the order of the computation load and the relation between the system variants, that is, the ratio of the two run times. The computation complexity metrics has to provide the correct ranking and correct proportionality of the computation load for the different parameter settings.

We compare the computation complexity between different configurations for the same data set. We assume, if the same data set is processed, then the same number of speaker models is trained, and then the same number of speaker probabilities is calculated. We assume further that the training time and likelihood calculation time increases linearly with the size of the data. The computation complexity is influenced by the following evaluated parameters: sampling frequency, time frame duration, and overlap of time frames. We introduce for each parameter a complexity factor. To calculate the overall design choice relative computation complexity the product of sampling frequency complexity factor, STFT complexity factor, and overlap complexity factor is taken.

The computation complexity is proportional to the sampling frequency. We define the sampling frequency complex-

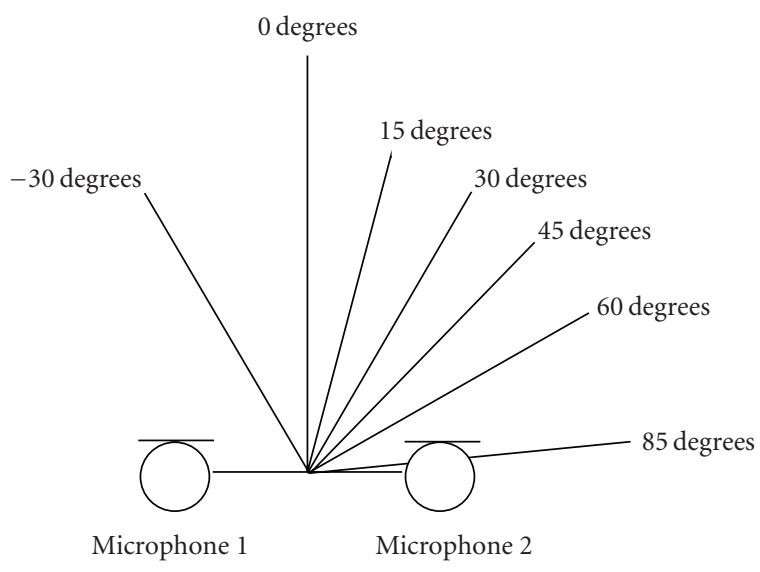

FIGURE 4: Microphone configuration and source directions.

ity factor for $8 \mathrm{kHz}$ as 1 and increase it proportionally to the sampling frequency, see Table 2.

The STFT complexity factor is defined as a weighted sum of the relative change of the number of processed data points plus the relative change of the processed time frames. As a first approximation, we set the weight for both to 0.5 . The resulting STFT complexity factor can be found in Table 3.

The overlap complexity factor is set to 1 when no overlapping occurs. If the time frames overlap by $50 \%$, then the number of frames and data points to process are doubled and the factor is set to 2 . If the time frames overlap by $75 \%$, the factor is set to 4 . If the time frames overlap by $85.5 \%$, the factor is set to 8 .

\section{EXPERIMENTS AND RESULTS}

We employ the experiments to show that the accuracy and relative computation complexity metrics introduced can be used to benchmark a two-step speaker separation and tracking system and to validate our system design. The experiments are based on 1 to 3 persons talking at fixed locations. The recordings are made in an office environment. Two microphones are placed on a table. A loudspeaker is placed $1 \mathrm{~m}$ away in front of the microphones. For the single-source experiment the loudspeaker is placed at $0,15,30,45,60,85$ degrees angles to the microphone axis, see Figure 4 . For the two-source experiment one loudspeaker was placed at 0 degrees and a second one at 30 degrees. For the three-source experiment one additional loudspeaker is placed at -30 degrees. The distance between the two microphones is $10 \mathrm{~cm}$, if not otherwise stated.

\subsection{Phase delay estimation (step (A))}

We compare delay estimations of DUET-PHAT, the GCCPHAT (GCC employing PHAT spectral weighting function), and the original DUET [17] algorithm. In the multisource case, we do not consider the GCC-PHAT estimations. We calculate the delay estimation distribution for each of the 6 


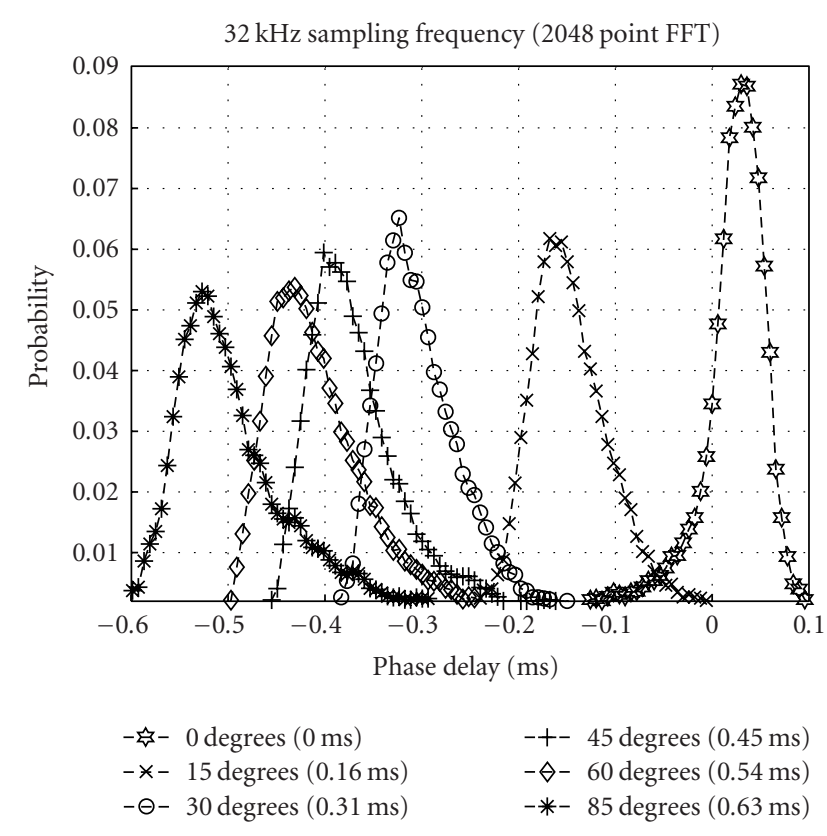

FIGURE 5: Smoothed probability distribution of the GCC-PHAT TDOA estimation. The microphone spacing is $20 \mathrm{~cm}$. The input signal is sampled at $32 \mathrm{kHz}$, and the microphone spacing is $20 \mathrm{~cm}$. The STFT length is 2048 points.

locations and for each sampling frequency and for each STFT length.

\subsubsection{GCC-PHAT}

The evaluation of the GCC-PHAT TDOA estimation showed four properties. First, the maximum of the TDOA estimation distribution is similar for the same time frame duration (e.g., $8 \mathrm{kHz}$ [sampling frequency]/512[STFT length], $16 \mathrm{kHz} / 1024$ and $32 \mathrm{kHz} / 2048$ ). Second, if the time frame duration is kept constant and the sampling frequency increases, the distribution gets narrower. Third, until a minimum time frame duration level is attained, that is, below 64 milliseconds for the selected configuration, the maximum of the distribution increases towards the true delay. Above this minimal time frame duration, the distribution maximum is constant. Fourth, Figure 5 shows that the distribution variance increases and that the difference between the true delay and the maximum of the distribution increases with the delay. Starting from 45 degrees, the difference is bigger than 0.05 millisecond. For our further evaluation, the GCC-PHAT delay estimation is employed as a reference. Based on the variance of the GCC-PHAT estimation, we set the tolerance to 0.025 millisecond for a $10 \mathrm{~cm}$ microphone spacing.

\subsubsection{Comparing GCC-PHAT, DUET, DUET-PHAT}

Figure 6 shows the F-measure of GCC-PHAT, DUET, and DUET-PHAT using two different low-pass cutoff frequencies

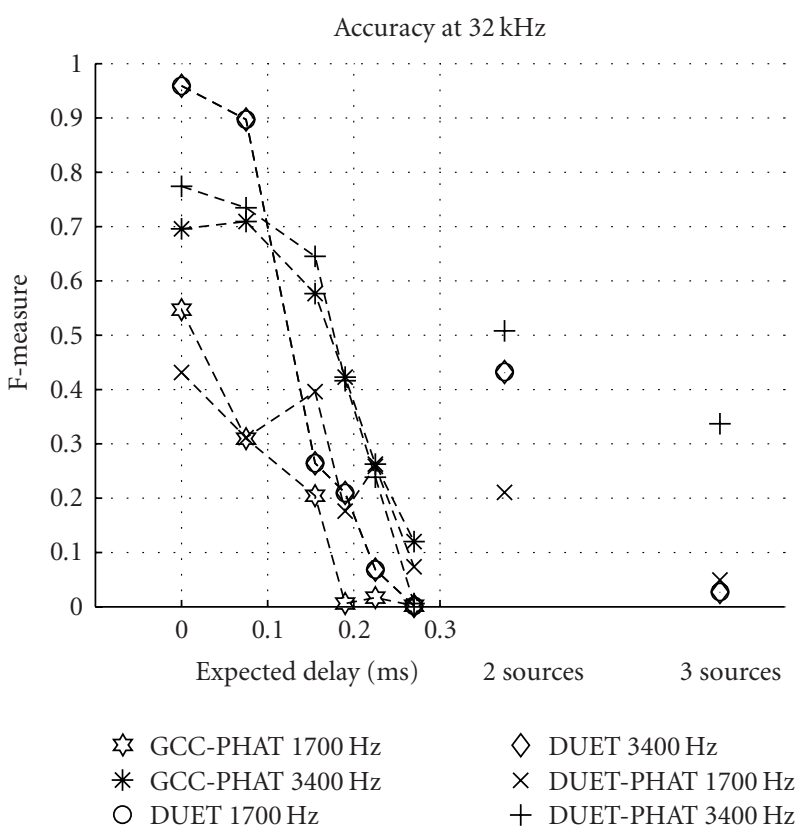

FIGURE 6: F-measure for the three different delay estimation algorithms. High-pass cutoff frequency $200 \mathrm{~Hz}$. Low-pass cutoff frequency is $1700 \mathrm{~Hz} / 3400 \mathrm{~Hz}$. Plotted are 6 delay locations and two multispeaker streams.

$\left(1700 \mathrm{~Hz}\left[f_{\text {crit }}\right]\right.$ and $\left.3400 \mathrm{~Hz}\left[2 \mathrm{x} f_{\text {crit }}\right]\right)$ and a high-pass cutoff frequency of $200 \mathrm{~Hz}$. The GCC-PHAT algorithm includes checks to ensure that only reliable TDOA estimations are reported. Consequently, whilst precision is high, recall is reduced.

For all approaches the accuracy declines with an increasing delay/angle and number of simultaneous sources. If the low-pass cutoff frequency is increased from $1700 \mathrm{~Hz}$ to $3400 \mathrm{~Hz}$, the GCC-PHAT and DUET-PHAT F-measure increases by at least $75 \%$. The two implementations benefit from the higher signal bandwidth as the spectral energy is normalized. The DUET algorithm is not affected as the signal energy is employed and as speech is expected to have maximum signal energy in the $250 \mathrm{~Hz}$ to $500 \mathrm{~Hz}$ band.

A comparison of the three implementations shows that DUET is best for small view angles but declines faster than the other two. DUET-PHAT is better than GCC-PHAT. For three simultaneous sources only DUET-PHAT has a Fmeasure above 0.3 .

We decided to employ the DUET-PHAT implementation as the performance is best in the multi-source scenarios. The F-measure declines also slower than for the DUET implementation, if the true delay increases (view angle).

\subsubsection{Microphone spacing}

A microphone spacing of $5 \mathrm{~cm}, 10 \mathrm{~cm}$, and $20 \mathrm{~cm}$ was evaluated. Reducing the microphone spacing increases $f_{\text {crit }}$ and consequently a higher signal bandwidth can be employed to estimate the source location. As the DUET-PHAT algorithm 


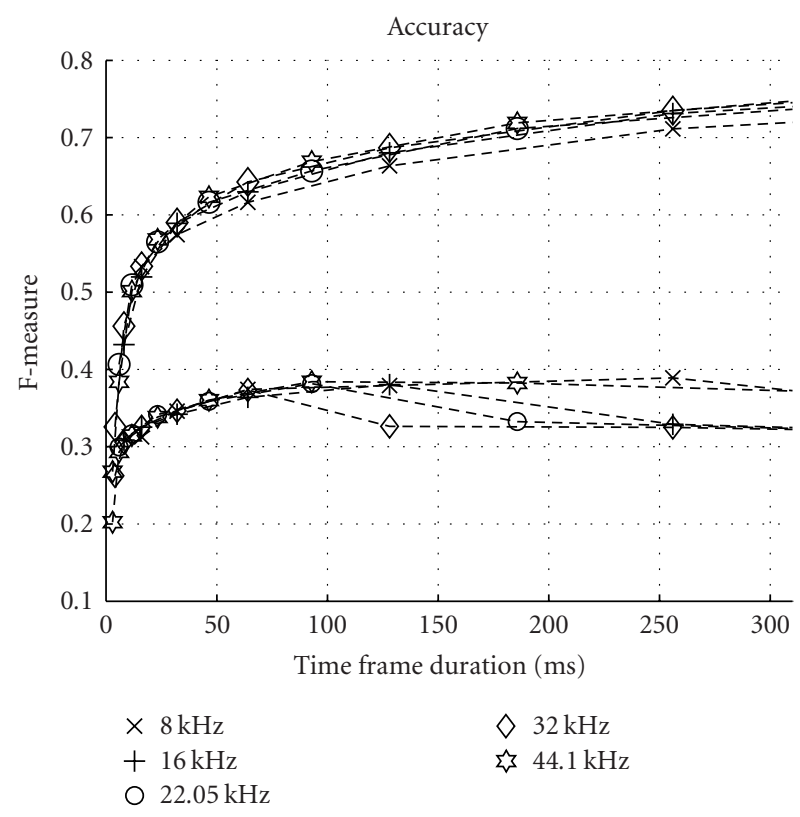

Figure 7: F-measure evaluated for 5 different sampling frequencies $(8 \mathrm{kHz}(\times), 16 \mathrm{kHz}(+), 22.05 \mathrm{kHz}(\circ), 32 \mathrm{kHz}(\diamond)$, and $44.1 \mathrm{kHz}$ $(\star))$ and a STFT length of $128,256,512,1024,2048$, and 4096 points. $\mathrm{X}$-axis is STFT length in $\mathrm{ms}$.

profits from a higher signal bandwidth, $5 \mathrm{~cm}$ showed best single-source accuracy followed by $10 \mathrm{~cm}$ and $20 \mathrm{~cm}$. In the multisource case a $5 \mathrm{~cm}$ spacing cannot separate more than two sources. $10 \mathrm{~cm}$ spacing shows best F-measure for two sources but a reduced value for three simultaneous sources compared to $20 \mathrm{~cm}$.

If the microphone spacing is reduced, the maximal delay values are reduced proportionally and small estimation fluctuations have a higher influence. In the single-source case these fluctuations are averaged as the number of data points is high. In the multisource case, the second or third source cannot be extracted as local maximum anymore. The sources are seen only as a small tip in the slope towards the global maximum, which could also be from noise.

The microphone spacing is therefore a tradeoff between signal bandwidth and delay estimation accuracy. The delay estimation accuracy is influenced, for example, by microphone noise and variation of the microphone spacing. The change of the delay by spacing variation has to be small compared to variations due to movements. For a microphone spacing of $10 \mathrm{~cm}$ a change by $0.5 \mathrm{~cm}$ is acceptable as similar changes by noise are observed.

\subsubsection{Time frame duration}

Figure 7 shows the F-measure for the speakers talking continuously at 30 degrees $(0.15$ millisecond $)$ and for three simultaneous sources. The time frame duration is varied from 3 milliseconds ( $44.1 \mathrm{kHz} / 128$ point STFT) to 510 milliseconds ( $8 \mathrm{kHz} / 4096$ point STFT, $32 \mathrm{kHz} / 16384)$.
In the single-source case, if the time frame-duration increases, the F-measure increases. In the multisource case, the F-measure maximum lies between 60 milliseconds and 130 milliseconds. Except for $8 \mathrm{kHz}$, where the maximum is at 256 milliseconds. The F-measure differs less then 3 percent compared to that measured at 128 milliseconds. The plotted F-measure is calculated with the minimal recall rate. If the average recall is considered, the maximum is moved towards longer time frame duration, the drop after the maximum is slower and the ascending slope of the F-measure is similar to the plotted one.

Time frame duration is a tradeoff between BSS accuracy and the assumption of speech quasistationarity. Blind source separation favors a time frame duration of 60 millisecods or longer. Aoki et al. [37] and Baeck and Zölzer. [38] presented best source separation for 100 milliseconds (maximal $\mathrm{W}$-disjoint orthogonality). On the other hand, in speech processing time frame durations of 30 milliseconds or below are typically employed.

Therefore, we decided to employ a time frame duration of 64 milliseconds for $8 \mathrm{kHz}, 16 \mathrm{kHz}$, and $32 \mathrm{kHz}$ and 93 milliseconds for $22.05 \mathrm{kHz}$ and $44.1 \mathrm{kHz}$ for our further experiments. Our speaker tracking experiments and the literature [41] show that under these conditions the sources can be separated and the quasistationarity assumption is still valid.

\subsubsection{Time frame overlap}

Table 4 shows for two locations ( 15 degrees and 30 degrees) and for three simultaneous sources that the time frame overlap has small influence on the location accuracy. The results for the tested sampling frequencies, the tested locations, and for simultaneous sources are similar to the one reported in Table 4. A system which only extracts location information would therefore be implemented with nonoverlap between the time frames to minimize the computation load.

\subsubsection{Sampling frequency}

If the sampling frequency is changed, that is, the frame duration and the overlap is kept constant, then the influence on the delay estimation accuracy is small, see Figure 8. For the source location detection we do not use the entire signal spectrum. Only signals in the frequency band $200 \mathrm{~Hz}$ to $3400 \mathrm{~Hz}$ are considered. As the time frame duration is equivalent to the frequency resolution, the number of points in the frequency band is independent of the sampling frequency and consequently the performance is similar. The slightly higher F-measure for $22.05 \mathrm{kHz}$ and $44.1 \mathrm{kHz}$ is due to the longer time segment.

\subsubsection{Conclusions}

To minimize computation load and maximize the performance, a low sampling frequency, nonoverlapping time frames, and a time frame duration between 50 milliseconds and 100 milliseconds should be used. The relative complexity is in the range from $0.62(8 \mathrm{kHz}$, no-overlap) to 36.15 
TABLE 4: The percentage gives the distance in which the time frame is moved. The values are recall/ precision.

\begin{tabular}{|c|c|c|c|c|c|}
\hline & $100 \%$ & $75 \%$ & $50 \%$ & $25 \%$ & $12.5 \%$ \\
\hline $08 \mathrm{kHz}$, STFT 512 , delay $0.08 \mathrm{~ms} \triangleq 15^{\circ}$ & $0.82 / 0.82$ & $0.83 / 0.81$ & $0.81 / 0.80$ & $0.82 / 0.82$ & $0.81 / 0.80$ \\
\hline $16 \mathrm{kHz}$, STFT 1024 , delay $0.08 \mathrm{~ms} \triangleq 15^{\circ}$ & $0.81 / 0.81$ & $0.83 / 0.83$ & $0.81 / 0.81$ & $0.82 / 0.81$ & $0.81 / 0.81$ \\
\hline $08 \mathrm{kHz}$, STFT 512 , delay $0.15 \mathrm{~ms} \triangleq 30^{\circ}$ & $0.69 / 0.57$ & $0.71 / 0.58$ & $0.68 / 0.56$ & $0.70 / 0.57$ & $0.68 / 0.57$ \\
\hline $16 \mathrm{kHz}$, STFT 1024 , delay $0.15 \mathrm{~ms} \triangleq 30^{\circ}$ & $0.69 / 0.57$ & $0.70 / 0.58$ & $0.70 / 0.58$ & $0.71 / 0.59$ & $0.68 / 0.56$ \\
\hline 3 streams $(-0.15 \mathrm{~ms} / 0.0 \mathrm{~ms} / 0.15 \mathrm{~ms}) 8 \mathrm{kHz}$, STFT 512 & $0.22 / 0.69$ & $0.22 / 0.71$ & $0.26 / 0.68$ & $0.23 / 0.69$ & $0.23 / 0.70$ \\
\hline 3 streams $(-0.15 \mathrm{~ms} / 0.0 \mathrm{~ms} / 0.15 \mathrm{~ms}) 16 \mathrm{kHz}$, STFT 1024 & $0.21 / 0.70$ & $0.22 / 0.70$ & $0.25 / 0.67$ & $0.22 / 0.69$ & $0.22 / 0.69$ \\
\hline
\end{tabular}

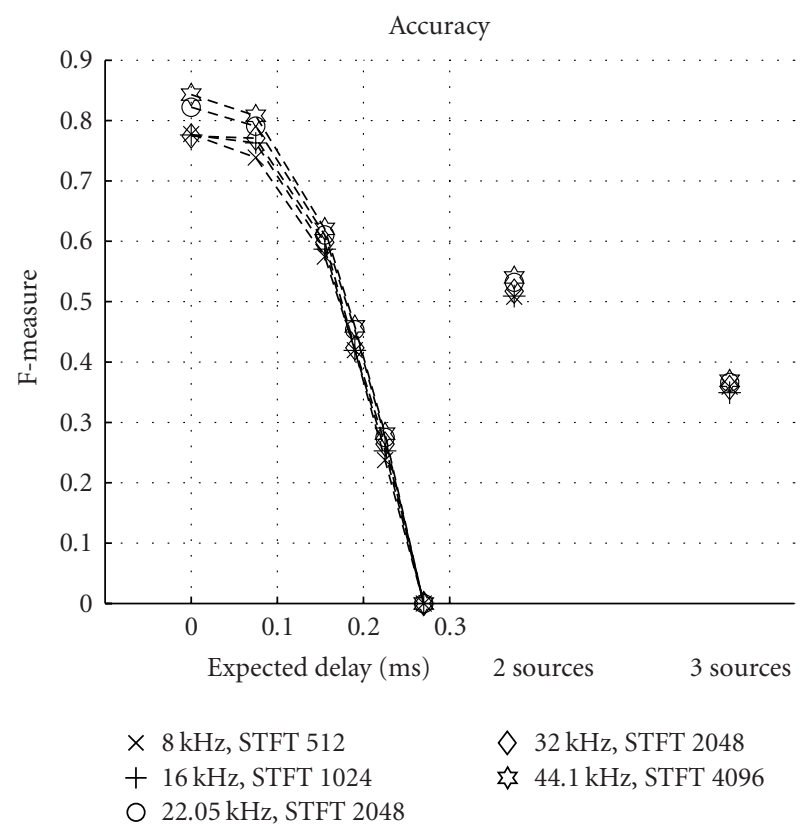

FIGURE 8: F-measure evaluated for constant time frame duration and 5 different sampling frequencies.

(44.1 kHz, $12.5 \%$ time frame shift). This outcome reduces the possible parameter combinations from 120 to $24(80 \%$ reduction).

\subsection{Speaker tracking (step $A+B)$}

The overall system performance is evaluated in two steps. First, the improvement of the source separation by the feedback loop is shown. And second, system recall and false rate are evaluated.

\subsubsection{Influence of the feedback}

Table 5 shows recall and precision with and without feedback loop. In the single-source case, if the delay is below 0.15 millisecond, then the BSS algorithm retrieves the correct location for $75 \%$ or more of the time segments. The feedback slightly increases the recall rate. Starting from 0.19 millisecond the BSS algorithm erroneously retrieves two sources instead of one. Precision is roughly halved compared to delays of 0.15 millisecond and below. Depending on the input signal either one or both locations are retrieved. The feedback adds the missed location to the estimation if the last segment has been classified as speech. Adding missed location increases the recall rate. The segment of the true location is more often classified as speech than the other one, this leads to increased precision. If no reliable source location is possible as for 0.27 millisecond delay, the feedback cannot improve the situation. In the multisource case the feedback adds delays of speaker locations which have not been detected and therefore recall is increased.

\subsubsection{System recall and false rate}

The evaluation of the blind source separation showed that speakers are detected in up to $80 \%$ of the cases if one individual is speaking and the view angle is smaller than 30 degrees. If the speakers are located at greater angles (more to the side) recall rapidly deteriorates. In the multisource case recall is about half of the single-source case. We first tested if a human can distinguish between individual speakers. The test subject observed that the filter process introduces a clicking noise and that acoustical changes are in some instances abrupt.

Table 6 reports the results for one speaker at 30 degrees and Table 7 for two simultaneous speakers. System recall, false rate, number of retrieved speakers, and thlike for which the results are achieved are stated in the table. Two simultaneous speakers give a lower system recall and a higher false rate than one speaker as the source separation introduce noise and acoustical changes. For three simultaneous speakers no speaker identification was possible due to interferences introduced by the separation.

Highest system recall and lowest false rate is shown for $8 \mathrm{kHz}$ sampling frequency. The performance difference between the sampling frequencies is significantly smaller for other data sets. The $16 \mathrm{kHz}$ sampling frequency accuracy is similar to higher sampling frequencies as the input signal is low-pass filtered at $7.5 \mathrm{kHz}$. For other experiments best accuracy has been observed for sampling frequencies other than $8 \mathrm{kHz}$.

For an autonomous system the threshold $t h_{\text {like }}$ has to be independent of the data set, experiment, and number of speakers. The experiments showed that the optimal threshold differs between data sets and experiments. We also observed an intraspeaker variability which leads in some instances to far more retrieved speakers than there are in the ground truth (e.g., $16 \mathrm{kHz}$, Table 6). 
TABLE 5: Recall/ precision for different locations. $16 \mathrm{kHz}$ sampling frequency. [200,3400] Hz bandpass. Step (A) does not include the knowledge of previous separation steps. Feedback does include location into the BSS step which has been classified as active location.

\begin{tabular}{|c|c|c|c|c|c|c|}
\hline & $0.00 \mathrm{~ms} \triangleq 0^{\circ}$ & $0.08 \mathrm{~ms} \triangleq 15^{\circ}$ & $0.15 \mathrm{~ms} \triangleq 30^{\circ}$ & $0.19 \mathrm{~ms} \triangleq 45^{\circ}$ & $0.23 \mathrm{~ms} \triangleq 60^{\circ}$ & $0.27 \mathrm{~ms} \triangleq 85^{\circ}$ \\
\hline Step (A) & $0.82 / 0.84$ & $0.77 / 0.77$ & $0.73 / 0.62$ & $0.59 / 0.35$ & $0.33 / 0.23$ & $0.01 / 0.01$ \\
\hline \multirow[t]{3}{*}{ Feedback } & $0.82 / 0.77$ & $0.81 / 0.77$ & $0.78 / 0.64$ & $0.72 / 0.38$ & $0.52 / 0.30$ & $0.01 / 0.01$ \\
\hline & \multicolumn{6}{|c|}{ Results for 2 and 3 simultaneous sources } \\
\hline & \multicolumn{3}{|c|}{$0 \mathrm{~ms}$ and $0.15 \mathrm{~ms}$} & \multicolumn{3}{|c|}{$-0.15 \mathrm{~ms}, 0 \mathrm{~ms}$, and $0.15 \mathrm{~ms}$} \\
\hline Step (A) & \multicolumn{3}{|c|}{$0.39 / 0.81$} & \multicolumn{3}{|c|}{$0.24 / 0.67$} \\
\hline Feedback & \multicolumn{3}{|c|}{$0.45 / 0.81$} & \multicolumn{3}{|c|}{$0.30 / 0.67$} \\
\hline
\end{tabular}

TABLE 6: System recall and false rate. Location $0.15 \mathrm{~ms} \triangleq 30$ degrees, single source, 4 speakers (1 female, 3 males).

\begin{tabular}{|c|c|c|c|c|c|}
\hline & $8 \mathrm{kHz} / 512$ & $16 \mathrm{kHz} / 1024$ & $22.05 \mathrm{kHz} / 2048$ & $32 \mathrm{kHz} / 2048$ & $44.1 \mathrm{kHz} / 4096$ \\
\hline Recall & 0.64 & 0.43 & 0.32 & 0.43 & 0.43 \\
\hline False rate & 0.36 & 0.55 & 0.64 & 0.51 & 0.47 \\
\hline Number of speakers & 3 & 15 & 6 & 16 & 11 \\
\hline$t h_{\text {like }}$ & 18 & 7 & 8 & 10.5 & 10 \\
\hline
\end{tabular}

TABLe 7: System recall and false rate. Two simultaneous sources, 4 speakers (2 females, 2 males).

\begin{tabular}{lcccccc}
\hline & $8 \mathrm{kHz} / 512$ & $16 \mathrm{kHz} / 1024$ & $16 \mathrm{kHz} / 1024$ & $22.05 \mathrm{kHz} / 2048$ & $32 \mathrm{kHz} / 2048$ & $44.1 \mathrm{kHz} / 4096$ \\
\hline Recall & 0.33 & 0.39 & 0.33 & 0.28 & 0.27 & 0.20 \\
False rate & 0.56 & 0.69 & 0.56 & 0.51 & 10 & 0.63 \\
Number of speakers & 5 & 5 & 3 & 8.5 & 10 & 0.59 \\
$t h_{\text {like }}$ & 12 & 7 & 4 & 8.5 & 3 \\
\hline
\end{tabular}

TABLE 8: Speaker confusion matrix. Two females (SP T1, SP T3) and 2 males (SP T2, SP T4) ground truth speakers. Bold represents the mapping between retrieved and true speakers.

\begin{tabular}{|c|c|c|c|c|c|c|c|c|c|}
\hline & \multicolumn{4}{|c|}{$\begin{array}{c}8 \mathrm{kHz} / 512, \text { delay } 0.08 \mathrm{~ms} \triangleq 15^{\circ} \\
\operatorname{rec}_{\text {sys }}=0.50, \text { fal }_{\text {sys }}=0.51, \text { dis }_{t h}=18\end{array}$} & \multicolumn{5}{|c|}{$\begin{array}{l}16 \mathrm{kHz} / 1024,2 \text { simultaneous sources } \\
\operatorname{rec}_{\text {sys }}=0.39, \mathrm{fal}_{\mathrm{sys}}=0.69, \mathrm{dis}_{t h}=7\end{array}$} \\
\hline & SP R1 & SP R2 & SP R3 & SP R4 & SP R1 & SP R2 & SP R3 & SP R4 & SP R5 \\
\hline SP T1 & 1.00 & 0.00 & 0.00 & 0.00 & 0.00 & 0.36 & 0.00 & 0.00 & 0.48 \\
\hline SP T2 & 0.27 & 0.52 & 0.10 & 0.10 & 0.00 & 0.05 & 0.00 & 0.04 & 0.80 \\
\hline SP T3 & 1.00 & 0.02 & 0.00 & 0.00 & 0.01 & 0.47 & 0.00 & 0.00 & 0.42 \\
\hline SP T4 & 0.88 & 0.00 & 0.00 & 0.00 & 0.00 & 0.27 & 0.00 & 0.05 & 0.63 \\
\hline
\end{tabular}

\subsubsection{Speaker confusion matrix}

Table 8 shows the confusion matrix for a single-source at 15 degrees and two simultaneous sources. In the single source case mostly SP R1 is retrieved. For SP T2, 52\% of the time SP $\mathrm{R} 2$ is retrieved.

In the multisource case, mainly SP R2 and SP R5 are retrieved. The SP R2 and SP R5 are not assigned to one location. SP R2 is retrieved for the first two minutes and SP R5 afterwards. To SP R1 and SP R3 only segments are assigned which have in total less than $1 \%$ of the ground truth speaking time. For the two simultaneous sources, the mapping between retrieved and ground truth speaker looks as follows: SP T1-SP R2, SP T2-SP R5, SP T3-SP R1, and SP T4-SP R4.

\subsubsection{Conclusion}

A feedback from the speaker tracking step to the BSS improves the location performance. The evaluation of the sys- tem recall, system false rate, and speaker confusion matrix showed that the identification step can be improved and the parameter cannot be fixed at this stage. To improve the identification step, the clicking noise introduced by the filtering process has to be reduced by means of incorporating speech properties. Additionally, the three metrics have been shown to be a valuable tool to judge the performance.

\section{CONCLUSION}

In this paper, we have presented a system that combines speaker separation and tracking in a two-step algorithm. The system addresses the speaker tracking problem also if overlaps between different sound sources exist. The system has been designed taking the constraints of a mobile environment into account, such as limited available system resources and dynamic acoustical parameters.

Additionally, we proposed a novel benchmark methodology to evaluate accuracy and computation complexity. Our 
benchmark has supported system design by reducing the number of three parameter tuples by $80 \%$ (from 120 to 24 tuples). Furthermore, our results support the case that feedback from the speaker tracking step to the blind source separation can benefit location accuracy by up to $20 \%$. We also found that system performance deteriorated with increasing delay (angle), and number of sources (BSS F-measure is reduced by each additional source by about $1 / 3$ ).

By reducing the employed signal bandwidth and weighting the signal spectrum the separation accuracy was improved compared to the standard DUET algorithm presented in [17]. We have additionally shown that for our implementation the blind source separation (based on delay estimation accuracy) is independent of sampling frequency but highly related to frame duration.

Issues that we did not consider include similar voices and the influence of the environment (e.g., background noise is different in a control room or outdoors). Once the identification accuracy issue has been resolved, we are optimistic that we will produce successful hardware implementations.

\section{REFERENCES}

[1] D. Moore, “The IDIAP smart meeting room," IDIAP-COM 07, IDIAP, 2002.

[2] C. Wooters, N. Mirghafori, A. Stolcke, et al., "The 2004 ICSISRI-UW meeting recognition system," in Lecture Notes in Computer Science, vol. 3361, pp. 196-208, January 2005.

[3] N. Kern, B. Schiele, H. Junker, P. Lukowicz, and G. Tröster, "Wearable sensing to annotate meeting recordings," Personal Ubiquitous Computing, vol. 7, no. 5, pp. 263-274, 2003.

[4] T. Choudhury and A. Pentland, "The sociometer: a wearable device for understanding human networks," in Proceedings of the Conference on Computer Supported Cooperative Work (CSCW'02), Workshop on Ad hoc Communications and Collaboration in Ubiquitous Computing Environments, New Orleans, La, USA, November 2002.

[5] S. Kwon and S. Narayanan, "A method for on-line speaker indexing using generic reference models," in Proceedings of the 8th European Conference on Speech Communication and Technology, pp. 2653-2656, Geneva, Switzerland, September 2003.

[6] M. Nishida and T. Kawahara, "Speaker model selection using Bayesian information criterion for speaker indexing and speaker adaptation," in Proceedings of the 8th European Conference on Speech Communication and Technology, pp. 18491852, Geneva, Switzerland, September 2003.

[7] L. Lu and H.-J. Zhang, "Speaker change detection and tracking in realtime news broadcasting analysis," in Proceedings of the 10th ACM International Conference on Multimedia, pp. 602610, Juan les Pins, France, December 2002.

[8] G. Lathoud, I. A. McCowan, and J.-M. Odobez, "Unsupervised location based segmentation of multi-party speech," in Proceedings of IEEE International Conference on Acoustics, Speech, and Signal Processing - Meeting Recognition Workshop (ICASSP-NIST '04), Montreal, Canada, May 2004, IDIAP-RR 04-14.

[9] M. Siracusa, L. P. Morency, K. Wilson, J. Fisher, and T. Darrell, "A multi-modal approach for determining speaker location and focus," in Proceedings of the International Conference on Multi-modal Interfaces (ICMI '03), pp. 77-80, Vancouver, BC, Canada, November 2003.
[10] J. Ajmera, G. Lathoud, and I. A. McCowan, "Clustering and segmenting speakers and their locations in meetings," Research Report IDIAP-RR 03-55, Dalle Molle Institute for Perceptual Articicial Intelligence (IDIAP), December 2003.

[11] C. Busso, S. Hernanz, C.-W. Chu, et al., "Smart room: participant and speaker localization and identification," in Proceedings of IEEE International Conference on Acoustics, Speech, and Signal Processing (ICASSP '05), vol. 2, pp. 1117-1120, Philadelphia, Pa, USA, March 2005.

[12] O. Amft, M. Lauffer, S. Ossevoort, F. Macaluso, P. Lukowicz, and G. Tröster, "Design of the QBIC wearable computing platform," in Proceedings of 15th IEEE International Conference on Application-Specific Systems, Architectures and Processors (ASAP '04), pp. 398-410, September 2004.

[13] S. Mann, "Wearable computing as means for personal empowerment," in 1st International Conference on Wearable Computing (ICWC '98), Fairfax, Va, USA, May 1998.

[14] A. Pentland, "Wearable intelligence," Scientific American, vol. 276, no. 1es1, 1998.

[15] E. Shriberg, A. Stolcke, and D. Baron, "Observations on overlap: findings and implications for automatic processing of multi-party conversation," in Poceedings of 7th European Conference on Speech Communication and Technology Eurospeech, vol. 2, pp. 1359-1362, Aalborg, Denmark, September 2001.

[16] R. Ferber, Information Retrieval, dpunkt, Germany, 2003.

[17] O. Yilmaz and S. Rickard, "Blind separation of speech mixtures via time-frequency masking," IEEE Transactions on Signal Processing, vol. 52, no. 7, pp. 1830-1847, 2004.

[18] S. Rickard, R. Balan, and J. Rosca, "Blind source separation based on space-time-frequency diversity," in Proceedings of 4th International Symposium on Independent Component Analysis and Blind Source Separation, pp. 493-498, Nara, Japan, April 2003.

[19] S. Rickard and Z. Yilmaz, "On the approximate W-disjoint orthogonality of speech," in Proceedings of the IEEE International Conference on Acoustics, Speech, and Signal Processing (ICASSP '02), vol. 1, pp. 529-532, Orlando, Fla, USA, May 2002.

[20] P. Aarabi and A. Mahdavi, "The relation between speech segment selectivity and source localization accuracy," in Proceedings of the IEEE International Conference on Acoustics, Speech, and Signal Processing (ICASSP '02), vol. 1, pp. 273-276, Orlando, Fla, USA, May 2002.

[21] S. Basu, S. Schwartz, and A. Pentland, "Wearable phased arrays for sound localization enhancement," in Proceedings of the IEEE International Symposium on Wearable Computing (ISWC '00), pp. 103-110, Atlanta, Ga, USA, 2000.

[22] A. Tritschler and R. Gopinath, "Improved speaker segmentation and segments clustering using the Bayesian information criterion," in Proceedings of the 6th European Conference on Speech Communication and Technology (EUROSPEECH '99), pp. 679-682, Budapest, Hungary, September 1999.

[23] L. Lu, H. Jiang, and H. J. Zhang, "A robust audio classification and segementation method," in Proceedings of the 9th ACM International Conference on Multimedia, pp. 203-211, Ottawa, Ontario, Canada, September-October 2001.

[24] V. Peltonen, J. Tuomi, A. Klapuri, J. Huopaniemi, and T. Sorsa, "Computational auditory scene recognition," in Proceedings of the IEEE International Conference on Acoustics, Speech, and Signal Processing (ICASSP '02), vol. 2, pp. 1941-1944, Orlando, Fla, USA, May 2002.

[25] E. Scheirer and M. Slaney, "Construction and evaluation of a robust multifeature speech/music discriminator," in Proceedings of the IEEE International Conference on Acoustics, Speech, 
and Signal Processing (ICASSP'97), vol. 2, pp. 1331-1334, Munich, Germany, April 1997.

[26] B. S. Atal, "Effectiveness of linear prediction characteristics of the speech wave for automatic speaker identification and verification," The Journal of the Acoustical Society of America, vol. 55, no. 6, pp. 1304-1312, 1974.

[27] G. Schwarz, "Estimating the dimension of a model," The Annals of Statistics, vol. 6, no. 2, pp. 461-464, 1978.

[28] P. Delacourt, D. Kryze, and C. Wellekens, "Speaker-based segmentation for audio data indexing," in Proceedings of the ESCA Tutorial and Research Workshop (ITRW'99). Accessing Information in Spoken Audio, pp. 78-83, Cambridge, UK, April 1999.

[29] M. Cettolo and M. Vescovi, "Efficient audio segmentation algorithms based on the BIC," in Proceedings of the IEEE International Conference on Acoustics, Speech, and Signal Processing (ICASSP '03), vol. 6, pp. 537-540, Hong Kong, April 2003.

[30] J. Ajmera, I. McCowanand, and H. Bourlard, "BIC revisited and applied to speaker change detection," in Proceedings of the IEEE International Conference on Acoustics, Speech, and Signal Processing (ICASSP '03), Hong Kong, April 2003.

[31] J. P. Campbell, "Speaker recognition: a tutorial," Proceedings of the IEEE, vol. 85, no. 9, pp. 1437-1462, 1997.

[32] D. A. Reynolds and R. C. Rose, "Robust text-independent speaker identification using Gaussian mixture speaker models," IEEE Transactions on Speech and Audio Processing, vol. 3, no. 1, pp. 72-83, 1995.

[33] M. Nishida and T. Kawahara, "Unsupervised speaker indexing using speaker model selection based on Bayesian information criterion," in Proceedings of the IEEE International Conference on Acoustics, Speech, and Signal Processing (ICASSP '03), vol. 1, pp. 172-175, Hong Kong, April 2003.

[34] T. Matsui and S. Furui, "Comparison of text-independent speaker recognition methods using VQ-distortion and discrete/continuous HMMs," in IEEE International Conference on Acoustics, Speech, and Signal Processing (ICASSP '92), vol. 2, pp. 157-160, San Francisco, Calif, USA, March 1992.

[35] F. Bimbot, J. Bonastre, C. Fredouille, et al., "A tutorial on textindependent speaker verification," EURASIP Jounral on Applied Signal Processing, vol. 2004, no. 4, pp. 430-451, 2004.

[36] H. Kawahara and T. Irino, "Exploring temporal feature representations of speech using neural networks," Tech. Rep. SP8831, IECIE, 1988.

[37] M. Aoki, M. Okamoto, S. Aoki, H. Matsui, T. Sakurai, and Y. Kaneda, "Sound source segregation based on estimating incident angle of each frequency component of input signals acquired by multiple microphones," Acoustical Science and Technology, vol. 22, no. 2, pp. 149-157, 2001.

[38] M. Baeck and U. Zölzer, "Real-time implementation of a source separation algorithm," in Proceedings of the 6th International Conference on Digital Audio Effects (DAFx'03), London, UK, September 2003.

[39] C. J. van Rijsbergen, Information retrieval, Butterworths, London, UK, 1979.

[40] U. Anliker, J. Beutel, and M. Dyer, "A systematic approach to the design of distributed wearable systems," IEEE Transactions on Computers, vol. 53, no. 8, pp. 1017-1033, 2004.

[41] J. L. He, L. Liu, and G. Palm, "A text-independent speaker identification system based on neural networks," in Proceedings of the International Conference on Spoken Language Processsing (ICSLP '94), pp. 1851-1854, Yokohama, Japan, September 1994.
U. Anliker received the Dipl.Ing. (M.S.) degree in electrical engineering from ETH Zurich, Switzerland, in 2000. In 2000, he joined the Wearable Computing Lab at the Electronics Laboratory at ETH Zurich where he is currently pursuing his Ph.D. degree. His research interests include low power wearable system design, blind source separation, and speaker identification systems.

J. F. Randall is an Independent Consultant supporting a number of academic projects. He was previously a Senior Research Fellow at the ETHZ (Eidgenssische Technische Hochschule Zurich), Switzerland. His Bachelor of Engineering with honours is from University of Wales College, Cardiff, and he holds a doctorate from the EPFL (Ecole Polytechnique Fédérale de Lausanne), Switzerland. He is a Char-

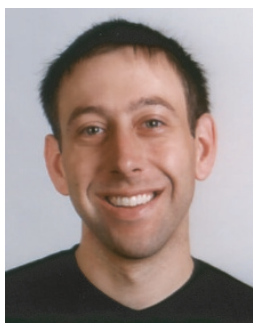
tered Engineer and a Member of the IEE and IEEE. He was the General Chair of the Second International Forum on Applied Wearable Computing in Zurich, Switzerland. His research interests include, but are not limited to, ambient energy power sources, context-aware wearable systems, autonomous systems, and human-computer interfaces. Any queries should be directed via jfrandall@iee.org.

G. Tröster received his M.S. degree from the Technical University of Karlsruhe, Germany, in 1978 and his Ph.D. degree from the Technical University of Darmstadt, Germany, in 1984, both in electrical engineering. He is a Professor and Head of the Electronics Laboratory, ETH Zurich, Switzerland. During the eight years at Telefunken Corporation, Germany, he was responsible for various national and international re-

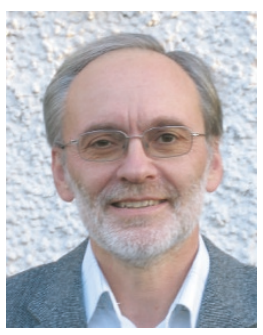
search projects focused on key components for ISDN and digital mobile phones. His field of research includes wearable computing, reconfigurable systems, signal processing, and electronic packaging. In 2000, he initiated the ETH Wearable Computing Lab as a Centre of Excellence. The Wearable Computing Group consisting of $15 \mathrm{Ph}$.D. students and additionally technical staff carries out research covering wired and wireless on-body connection, reconfigurable wearable computing platform, gesture recognition using miniaturized cameras, focus-free retinal displays, context recognition comprising the design of microsensor networks, low-power signal preprocessing, smart textiles, and algorithms for feature extraction and classification. Gerhard Tröster authored and coauthored more than 150 articles, and holds five patents. In 1997, he cofounded the spin-off company u-blox AG. 